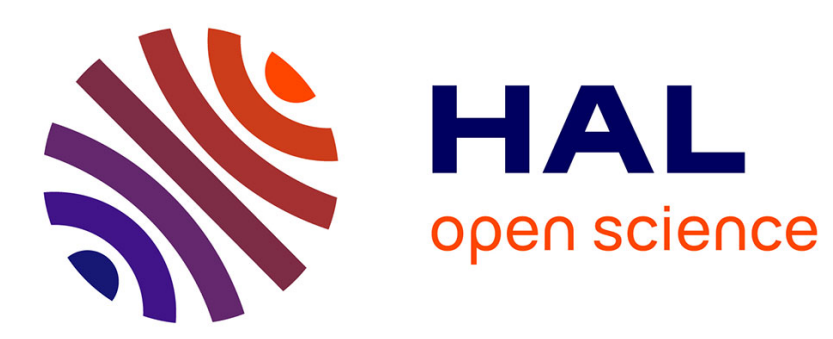

\title{
Design and performances evaluation of new Costas-based radar waveforms with pulse coding diversity
}

\author{
Nadjah Touati, Charles Tatkeu, Thierry Chonavel, Atika Rivenq
}

\section{To cite this version:}

Nadjah Touati, Charles Tatkeu, Thierry Chonavel, Atika Rivenq. Design and performances evaluation of new Costas-based radar waveforms with pulse coding diversity. IET Radar Sonar and Navigation, 2015, 15p. 10.1049/iet-rsn.2014.0450 . hal-01213558

\section{HAL Id: hal-01213558 \\ https://hal.science/hal-01213558}

Submitted on 8 Oct 2015

HAL is a multi-disciplinary open access archive for the deposit and dissemination of scientific research documents, whether they are published or not. The documents may come from teaching and research institutions in France or abroad, or from public or private research centers.
L'archive ouverte pluridisciplinaire HAL, est destinée au dépôt et à la diffusion de documents scientifiques de niveau recherche, publiés ou non, émanant des établissements d'enseignement et de recherche français ou étrangers, des laboratoires publics ou privés. 


\title{
Design and Performances Evaluation of New Costas-Based Radar Waveforms with Pulse Coding Diversity
}

\author{
Nadjah TOUATI, Charles TATKEU, Thierry CHONAVEL， and Atika RIVENQ \\ Univ Lille Nord de France, IFSTTAR, Télécom Bretagne, UMR CNRS 6285, Lab-STICC, \\ IEMN-DOAE Université de Valenciennes \\ \{nadjah.touati,charles.tatkeu\}@ifsttar.fr \\ thierry.chonavel@telecom-bretagne.eu \\ atika.menhaj@univ-valenciennes.fr
}

May 23, 2015

\begin{abstract}
Costas codes are a variant of pulse compression waveforms, largely studied for their attractive time-frequency properties. Their «thumbtack-like » ambiguity function makes them highly suitable for delay and Doppler estimation, in radar and sonar applications. However, this behavior depends heavily on the length of the code: the improvement in delay-Doppler resolutions and ambiguity function sidelobes level needs an increase in the size of the code. In this paper, designs that allow good performance without increasing the size of the code are proposed. They are based on a modification of Costas Codes by widening frequency separation between hops and replacing rectangular pulses by other waveforms. This will lead to a removal of autocorrelation function ACF grating lobes that normally appear when frequency separation is increased. The originality of the work lies in the proposal of diversified pulse waveforms, such as phase codes, Slepian sequences and other Costas codes, to encode main Costas pulses. A performance comparison of the proposed approaches is supplied. Such waveforms could also be of interest for applications where waveform diversity is desired.
\end{abstract}

\section{Introduction}

Pulse compression is a technique used in radar systems to increase both range resolution and signal to noise ratio. It consists in modulating the transmitted pulse and then correlating the received signal with a replica of the transmitted pulse [1]. Frequency and phase modulated waveforms are frequently used in pulse compression [2]. Time-frequency codes are a variant of frequency modulated signals that show high time-frequency resolution. Examples of such codes are Costas codes or Costas arrays[3]. They were introduced as a means of improving performance of radar and sonar systems. Indeed, Costas codes have near ideal range and Doppler sidelobe behavior, providing both unambiguous doppler and range information.

A Costas code consists of an $M \times M$ time-frequency code with a randomlike evolution. An example, for $\mathrm{M}=8$, is supplied in Fig 1. The main feature of these codes is that a single frequency $f_{m}$, used only once, is emitted in each time interval $t_{p}$. These 
Touati, Nadjah; Tatkeu, Charles; Chonavel, Thierry; Rivenq, Atika:

Design and performances evaluation of new Costas-based radar waveforms with pulse coding diversity',

IET Radar, Sonar and Navigation, 2015, DOI: 10.1049/iet-rsn.2014.0450

codes are designed so as to minimize coincidences between the signal and its time and frequency shifted copies. This property can be evaluated through the ambiguity function $\mathrm{AF}|\chi(\tau, \nu)|$. Ideally, it should be a 2D dirac of maximized energy in $(\tau, \nu)=(0,0)$ and zero elsewhere. The properties of Costas codes allow better approximation of the ideal ambiguity function AF, as shown in Fig 2 , where the AF of the Costas code $[2,6,3,8,7,5,1,4]$ is plotted. The thumbtack behavior can be clearly seen and all sidelobes have amplitude $1 / M$, except a few close to the origin, which are a little bit higher.

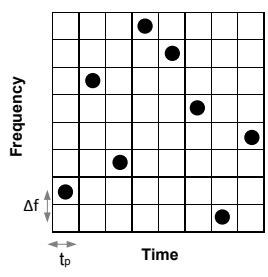

Figure 1: Example of a Costas code of size $\mathrm{M}=8:[2,6,3,8,7,5,1,4]$

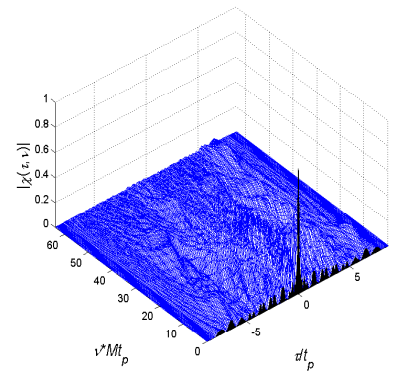

Figure 2: Ambiguity function of the Costas code $[2,6,3,8,7,5,1,4]$.

The literature proposes algorithms for building such codes [4],[5],[6]. A more general approach consists in searching among all possible sequences of length $\mathrm{M}$ (all M! sequences), those which follow Costas properties by using the difference matrix method [7]. In this paper, we are interested in studying modified versions of Costas signals. In the literature, some works focused on the modification of those signals in order to improve peak to sidelobe ratio (PSLR) in the autocorrelation function (ACF). A train of staggered Costas signals was proposed in [8], in order to allow AF improvement. In the same spirit, an overlaying of orthogonal codes in a train of Costas pulses was proposed in [9]. In [10],[11],[12], pulse modulation of Costas frequency hops was introduced, using phase codes and complementary sequences. However, few works study Costas signals beyond the orthogonality condition which requires that frequency separation $\Delta f$ should be at most equal to the inverse of pulse duration $t_{p}$ (i.e. $t_{p} \Delta f=1$ ). In [13], frequency modulation was introduced to perform modification of Costas codes when frequency separation is increased $\Delta f>\frac{1}{t_{p}}$. This widening is interesting since the overall time-bandwidth product could be achieved without increasing the Costas code size. In this case, the frequency modulation of Costas pulses allows the lowering of autocorrelation function grating lobes that appear when the frequency separation is increased. The work in this paper fits in this specific theme, which is waveform design based on modified Costas signals. Novel modified Costas designs are proposed where the $\Delta f>\frac{1}{t_{p}}$ case, which offers more flexibility to design such signals, is considered. The synthesized waveforms are based on Costas codes combined to pulse waveforms of different natures. They achieve good autocorrelation properties with lowered grating lobes, when design conditions are respected. The originality of the proposed approaches lies in the diversity introduced by using pulse waveforms of different natures. This can be useful, for instance, for multiple radars systems or multi-user purposes.

The paper is organized as follows. In section II, construction constraints for Costas codes and the modification principle are introduced. The state-of-the-art method for Costas signals modification using Linear Frequency Modulated LFM pulses is also presented. In section III, designs based on different pulse waveforms are proposed. For each case, the relevant parameters that allow a minimum sidelobe combination are defined. In section IV, a method for lowering the first Costas sidelobe is proposed based on 
previous results. In section $\mathrm{V}$, a performance comparison of the previous approaches is proposed.

\section{Costas signal modification}

\subsection{Orthogonality constraint and modification principle}

The complex envelope $x(t)$ of the Costas code $\left[a_{m}\right]_{m=0: M-1}$ is given by [2]:

$$
x(t)=\sum_{m=0}^{M-1} u_{m}\left(t-m t_{p}\right)
$$

where

$$
\begin{array}{r}
u_{m}(t)=e^{\left(j 2 \pi f_{m} t\right)} \times \operatorname{Rect}\left(\frac{t}{t_{p}}\right) \\
\text { and } \operatorname{Rect}\left(\frac{t}{t_{p}}\right)=\left\{\begin{array}{c}
1 \text { if } 0 \leq t \leq t_{p} \\
0 \text { elsewhere }
\end{array}\right.
\end{array}
$$

The design condition $\Delta f=\frac{1}{t_{p}}$ forces signal frequencies $f_{m}(m=0: M-1)$ to be multiples of $\frac{1}{t_{p}}$ that is, $f_{m}=\frac{a_{m}}{t_{p}}$. This condition guarantees the orthogonality among Costas pulses and hence low AF sidelobes. However, the orthogonality condition stated before is sometimes restrictive. For example, in some applications, signal duration is limited by radar measurement ambiguities and the bandwidth must be swept in minimum time. If a quick scan is needed, $\Delta f$ must be increased beyond the inverse of pulse duration $t_{p}$, namely $\Delta f>\frac{1}{t_{p}}$. The frequency in the $m^{t h}$ pulse $f_{m}$ changes according to the new $t_{p} \Delta f$ product: $f_{m}=a_{m} \Delta f=\frac{a_{m}}{t_{p}} \times t_{p} \Delta f$. However, increasing the frequency spacing while keeping rectangular pulses is not convenient. From a spectral point of view, the frequency spacing is increased but occupied by waveforms that do not fill all this spacing. Furthermore, as the orthogonality condition is not satisfied, grating lobes will appear around the mainlobe of the ACF. It was shown in [13] that around the mainlobe $\left(|\tau|<t_{p}\right)$, the ACF $R\left(\frac{\tau}{t_{p}}\right)$ is roughly the product of $R_{1}\left(\frac{\tau}{t_{p}}\right)$ the ACF of the rectangular pulse that constitutes the Costas hops and $R_{2}(\tau)$ the grating lobes distribution function. Hence, for $|\tau|<t_{p}$ :

$$
\left|R\left(\frac{\tau}{t_{p}}\right)\right| \approx\left|R_{1}\left(\frac{\tau}{t_{p}}\right)\right| \cdot\left|R_{2}\left(\frac{\tau}{t_{p}}\right)\right| \text { where }\left|R_{2}\left(\frac{\tau}{t_{p}}\right)\right|=\left|\frac{\sin \left(M \pi t_{p} \Delta f \frac{\tau}{t_{p}}\right)}{\sin \left(\pi t_{p} \Delta f \frac{\tau}{t_{p}}\right)}\right|
$$

Grating lobes are caused by maxima of $R_{2}(\tau)$ that appear at $\tau(k)=\frac{k}{t_{p} \Delta f} t_{p}$ where $k=0, \pm 1, \pm 2 \cdots \pm$ floor $\left(t_{p} \Delta f\right)$. In the case of rectangular pulses, $R_{1}\left(\frac{\tau}{t_{p}}\right)$ has a triangular shape and $R\left(\frac{\tau}{t_{p}}\right)$ shows equally spaced, linearly decreasing grating lobes, as shown in Fig 3, for the Costas code $[2,6,3,8,7,5,1,4]$ and $\Delta f=\frac{5}{t_{p}}$. Based on the above considerations, a change in Costas pulse waveforms must be operated in order to shape pulse ACF $R_{1}\left(\frac{\tau}{t_{p}}\right)$ in such a way that, when combined with $R_{2}\left(\frac{\tau}{t_{p}}\right)$, grating lobes are lowered.

The authors of [13] proposed nullifying them using Linear Frequency Modulated LFM pulses instead of rectangular ones. In this paper, alternative pulse waveforms with optimized parameters are proposed. The principle of modification of Costas signals is shown in Fig 4 where rectangular pulses are replaced by other pulse waveforms with complex envelope $A(t)$ of duration $t_{p}$ and a $3 \mathrm{~dB}$ bandwidth $B_{p}$. Hence, each frequency hop $f_{m}$ is modulated by the novel pulse waveform $A(t)$. The complex enveloppe of modified 

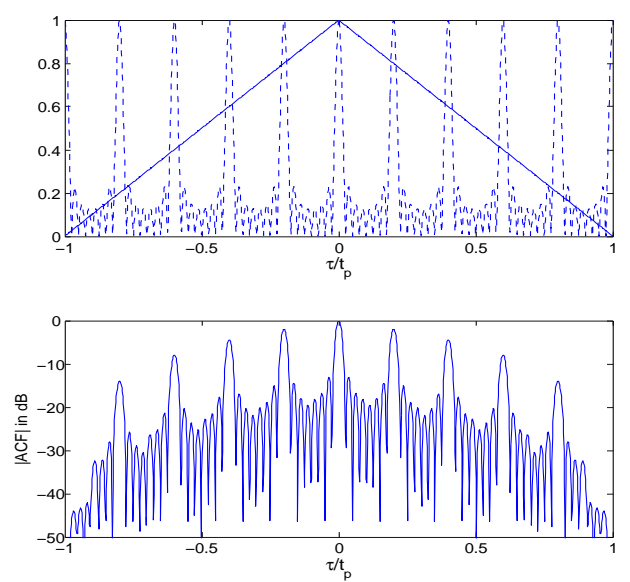

Figure 3: ACF of the Costas code $[2,6,3,8,7,5,1,4]$ in $|\tau|<t_{p}$, for $t_{p} \Delta f=5$ (Grating lobes), top: continuous $\left|R_{1}\left(\frac{\tau}{t_{p}}\right)\right|$, dashed $\left|R_{2}\left(\frac{\tau}{t_{p}}\right)\right|$ bottom: $\left|R\left(\frac{\tau}{t_{p}}\right)\right|$

Costas signals is then given by:

$$
x(t)=\sum_{m=0}^{M-1} u_{m}\left(t-m t_{p}\right)
$$

where

$$
u_{m}(t)=e^{\left(j 2 \pi f_{m} t\right)} \times A(t)
$$

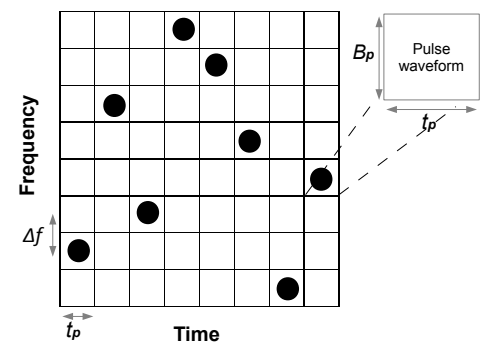

Figure 4: Costas signal modification principle.

Basic Costas signals will be the special case $A(t)=\operatorname{Rect}\left(\frac{t}{t_{p}}\right)$. The discussed pulse waveforms will be detailed in their dedicated parts. The design principle is based on ACF optimization by builiding combinations that guarantee grating lobe lowering. We extend the study to all the delay-Doppler behaviour by studying the $\mathrm{AF}$ of modified Costas signals $\chi(\tau, \nu)$. It is derived using results on $\mathrm{AF}$ of basic Costas signals, which is given by [3]:

$$
\begin{aligned}
\chi(\tau, \nu) & =\int_{-\infty}^{+\infty} x^{*}(t) x(t-\tau) e^{j 2 \pi \nu t} d t \\
\chi(\tau, \nu) & =\sum_{m=0}^{M-1} e^{+j 2 \pi m \nu t_{p}}\left[\Phi_{m m}(\tau, \nu)+\sum_{n=0, n \neq m}^{M-1} \Phi_{m n}\left(\tau-(m-n) t_{p}, \nu\right)\right]
\end{aligned}
$$

where $\Phi_{m n}(\tau, \nu)$ is defined in $|\tau| \leq t_{p}$ by:

$$
\Phi_{m n}(\tau, \nu)=\int_{-\infty}^{+\infty} u_{m}^{*}(t) u_{n}(t-\tau) e^{j 2 \pi \nu t} d t,|\tau| \leq t_{p}
$$


For modified Costas signals, $u_{m}(t)=A(t) \times e^{\left(j 2 \pi f_{m} t\right)}$. Hence, for $|\tau| \leq t_{p}, \Phi_{m n}(\tau, \nu)$ is given by:

$$
\begin{aligned}
& \Phi_{m n}(\tau, \nu)=\int_{-\infty}^{+\infty} A^{*}(t) e^{-j 2 \pi f_{m} t} A(t-\tau) e^{j 2 \pi f_{n}(t-\tau)} e^{j 2 \pi \nu t} d t \\
& \Phi_{m n}(\tau, \nu)=e^{-j 2 \pi f_{n} \tau} \int_{-\infty}^{+\infty} A^{*}(t) A(t-\tau) e^{j 2 \pi\left(\nu+\left(f_{n}-f_{m}\right)\right) t} d t \\
& \Phi_{m n}(\tau, \nu)=e^{-j 2 \pi f_{n} \tau} \chi_{A}\left(\tau, \nu+\left(f_{n}-f_{m}\right)\right),|\tau| \leq t_{p}
\end{aligned}
$$

where $\chi_{A}(\tau, \nu)$ is the AF of the pulse waveform. Replacing Eq 6 in Eq 4, we find the overall AF of modified signals:

$$
\begin{array}{r}
\chi(\tau, \nu)=\sum_{m=0}^{M-1} e^{+j 2 \pi m \nu t_{p}}\left[\chi_{A}(\tau, \nu) e^{-j 2 \pi f_{m} \tau}+\right. \\
\left.\sum_{n=0, n \neq m}^{M-1} \chi_{A}\left(\tau-(m-n) t_{p}, \nu+\left(f_{n}-f_{m}\right)\right) e^{-j 2 \pi f_{n}\left(\tau-(m-n) t_{p}\right)}\right]
\end{array}
$$

In [3], it was stated that around the mainlobe $|\tau| \leq t_{p}$, the AF mainly depends on the first term of the sum in Eq 7 . Hence, the overall $\mathrm{AF}$ is related to the pulse waveform $\mathrm{AF} \chi_{A}(\tau, \nu)$ as follows:

$$
\chi(\tau, \nu) \approx \chi_{A}(\tau, \nu) \sum_{m=0}^{M-1} e^{+j 2 \pi\left(m \nu t_{p}-f_{m} \tau\right)},|\tau| \leq t_{p}
$$

According to Eq 8, we can establish that the pulse wavefrom AF has a capital contribution to shape the AF of modified signals. This can be seen more specifically in the zero-Doppler and zero-delay cuts. Noting that $\sum_{m=0}^{M-1} e^{j \alpha m}=\frac{\sin \left(\frac{M \alpha}{2}\right)}{\sin \left(\frac{\alpha}{2}\right)} e^{j(M-1) \frac{\alpha}{2}}$ and $f_{m}=a_{m} \Delta f$, we get the zero-Doppler cut (autocorrelation function ACF) magnitude $\left|R\left(\frac{\tau}{t_{p}}\right)\right|=|\chi(\tau, 0)|$ in Eq 2 according to pulse $\mathrm{ACF}\left|R_{1}\left(\frac{\tau}{t_{p}}\right)\right|=\left|\chi_{A}(\tau, 0)\right|$. Note also that the frequency order $a_{m}$ does not affect the zero-Doppler cut in the mainlobe region. Similarly, the zero delay cut is also derived:

$$
\begin{aligned}
& |\chi(\tau, 0)| \approx\left|\chi_{A}(\tau, 0)\right|\left|\frac{\sin (M \pi \tau \Delta f)}{\sin (\pi \tau \Delta f)}\right|,|\tau| \leq t_{p} \\
& |\chi(0, \nu)| \approx\left|\chi_{A}(0, \nu)\right|\left|\frac{\sin \left(M \pi \nu t_{p}\right)}{\sin \left(\pi \nu t_{p}\right)}\right|,|\tau| \leq t_{p}
\end{aligned}
$$

The lowering of grating lobes results from the relative shapes $R_{1}\left(\frac{\tau}{t_{p}}\right)$ and $R_{2}\left(\frac{\tau}{t_{p}}\right)$. In order to achieve the grating lobes mitigation with minimum bandwidth consumption, $3 \mathrm{~dB}$ bandwidth $B_{p}$ of pulse waveforms must also be considered. To avoid several periods of $R_{2}\left(\frac{\tau}{t_{p}}\right)$ falling into the main lobe of $R_{1}\left(\frac{\tau}{t_{p}}\right)$, the first grating lobe position $\left(\tau=\frac{1}{\Delta f}\right)$ should be farther than the first null of $R_{1}\left(\frac{\tau}{t_{p}}\right)$ 
$\tau_{\text {first-null }}$ that is related to the inverse of pulse waveform bandwidth $B_{p}$ :

$$
\frac{1}{B_{p}} \leq \frac{1}{\Delta f} \Rightarrow t_{p} B_{p} \geq t_{p} \Delta f
$$

In addition to the first grating lobes elimination, this condition also guarantees a good spectral occupancy, since the pulse waveform bandwidth is at least equal to the frequency spacing. In practice, it is preferable to choose $B_{p}$ that fulfills Eq 10 while guaranteeing overlap ratio $\frac{B_{p}}{\Delta f}$ as little as possible. Before introducing designed waveforms based on these considerations, we recall, in the next section, the state-of-the-art method for Costas codes modification that involves LFM waveforms.

\subsection{Costas signals with LFM pulses}

A conventional method for grating lobes suppression in Costas signals consists in replacing rectangular pulses by LFM pulses with bandwidth $B_{p}$, that is setting $A(t)=\operatorname{Rect}\left(\frac{t}{t_{p}}\right) e^{j \pi\left(\frac{B_{p}}{t_{p}}\right) t^{2}}$ [13], with the following constraints for LFM bandwidth and Costas frequencies spacing:

$$
t_{p} \Delta f=\frac{4 m-n}{2 m-n} \text { and } t_{p} B_{p}=\frac{(4 m-n)^{2}}{2(2 m-n)} \text { where } m, n \in \mathbb{N}
$$

In Fig 5, an example of Costas signal with size $M=8$ coded with LFM pulses is shown, when $\left(t_{p} \Delta f, t_{p} B_{p}\right)$ is equal to $(7,24.5)$. Grating lobes are significantly lowered by the LFM ACF. Since grating lobes positions do not depend on Costas code size $M$, the designs conditions are still valid for other Costas sizes.
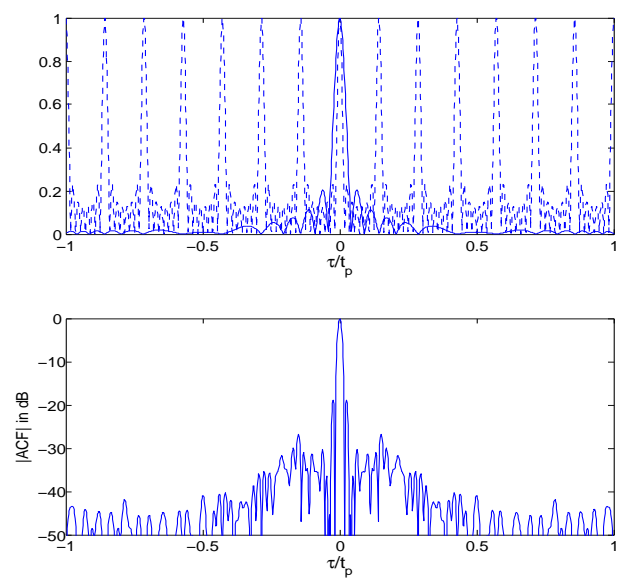

Figure 5: ACF of Costas-LFM signal with $\left(t_{p} \Delta f, t_{p} B_{p}\right)=(7,24.5),(m, n)=(3,5)$ top: continuous $\left|R_{1}\left(\frac{\tau}{t_{p}}\right)\right|$, dashed $\left|R_{2}\left(\frac{\tau}{t_{p}}\right)\right|$, bottom: $\left|R\left(\frac{\tau}{t_{p}}\right)\right|$

Relationships in Eq 11 are derived to position the first two grating lobes at two nulls of Pulse ACF [14]. However, for fixed $t_{p} \Delta f$, this sometimes gives rise to very high values of $t_{p} B_{p}$. For example, when $t_{p} \Delta f=6$, the minimmum value for $t_{p} B_{p}$ is 36 : $(m, n)=(5,8)$. This causes high bandwidth overflow $\left(\frac{B_{p}}{\Delta f}=6\right)$. It is proposed to alleviate the constraint by just coinciding the first grating lobe with a null of the LFM's ACF, leading thus to $\pi B_{p} \frac{k}{\Delta f}\left(1-\frac{k}{t_{p} \Delta f}\right)=m \pi$ [14] where $k$ is the grating lobe order and $m$ the LFM's ACF null order. Hence, when the $m^{t h}$ null of the LFM's ACF coincides with the first grating lobe $(k=1)$, we have: 


$$
t_{p} B_{p}=m \frac{\left(t_{p} \Delta f\right)^{2}}{\left(t_{p} \Delta f-1\right)}, m \in \mathbb{N}
$$

With this approach, the other grating lobes are also lowered, to some extent. Bandwidth overflow values are significantly lowered: in the previous example $t_{p} \Delta f=6$, the minimal required $t_{p} B_{p}$ is now equal to $t_{p} B_{p}=7.2$, and $\frac{B_{p}}{\Delta f}=1.2$. Some valid configurations of the Costas-LFM combinations, using Eq 12 are presented in Table 1. The choice of $m$ allows us to reconcile lowered grating lobes and limited bandwidth overflow.

Table 1: Example of valid configurations of Costas-LFM combination (Costas code $M=8$ )

\begin{tabular}{|c|c|c|c||c|c|c|c|}
\hline$m$ & $t_{p} \Delta f$ & $t_{p} B_{p}$ & $\frac{B_{p}}{\Delta f}$ & $m$ & $t_{p} \Delta f$ & $t_{p} B_{p}$ & $\frac{B_{p}}{\Delta f}$ \\
\hline 1 & 2 & 4 & 2 & 3 & 6 & 21.6 & 3.6 \\
2 & 2 & 8 & 4 & 2 & 7 & 16.33 & 2.33 \\
2 & 3 & 9 & 3 & 3 & 7 & 24.5 & 3.5 \\
3 & 3 & 13.5 & 4.5 & 2 & 8 & 18.26 & 2.29 \\
2 & 4 & 10.66 & 2.66 & 3 & 8 & 27.43 & 3.43 \\
3 & 4 & 16 & 4 & 2 & 9 & 20.25 & 2.25 \\
2 & 5 & 12.5 & 2.5 & 3 & 9 & 30.75 & 3.38 \\
3 & 5 & 18.75 & 3.75 & 2 & 10 & 22.22 & 2.22 \\
2 & 6 & 14.4 & 2.4 & 3 & 10 & 33.33 & 3.33 \\
\hline
\end{tabular}

This design is based on well-known and widely-used LFM chirps. It is well adapted for a single user context but it shows limitations for multi-user applications. Indeed, when multi-user waveforms are needed, the autocorrelation properties must remain acceptable, while providing the lowest possible cross-correlations. This can be achieved by coding differently Costas pulses among different users. Hence, we are interested in other waveforms that introduce more diversity and a large choice among Costas pulse waveforms, thus providing more flexibility for multi-user adaptation.

\section{Proposed designs}

\subsection{Costas signals with phase coded pulses}

In [15], we have shown that coding pulses, with codes that have good autocorrelation properties, significantly lowers the grating lobes level. The complex envelope of pulse waveforms will be: $A(t)=\sum_{l=0}^{L-1} q_{l} \operatorname{Rect}\left(\frac{t-l t_{c}}{t_{c}}\right)$ where $t_{c}=\frac{t_{p}}{L}$ and $\left[q_{l}\right]_{l=0: L-1}$ is the code sequence. For a phase code $t_{p} B_{p}=L$ and the condition in Eq 10 will depend on the length of the pulse phase code:

$$
L \geq t_{p} \Delta f
$$

Either binary phase codes, in which the phase elements are restricted to 0 or $\pi$, or polyphase codes, in which there is no restriction on phase increments, can be considered. Probably the most famous family of binary phase codes are Barker codes [16]. Several wellknown polyphase codes are derived from the phase history of LFM pulses. Examples of such codes are Frank codes, P1 and P2 codes, which are only available when $L=k^{2}$ [17] and Zadoff-chu codes, P3 and P4 codes, which are defined for any arbitrary length $L$ [18]. For example, a P3 phase code of length $L$ is given by $\left[q_{l}=e^{\phi_{l}}\right]_{l=0: L-1}$ where $\phi_{l}=\frac{2 \pi}{L} \frac{(l)^{2}}{2}$. An example of a phase coded Costas signal is provided in Fig 6 for a P3 code of length $L=4$. In this case, the ACF nulls of the P3 code of length $L=4$ coincide exactly 
with grating lobes positions and eliminate them. This becomes less obvious when $L$ and $t_{p} \Delta f$ are increased. Then, we try to coincide a maximum of nulls with grating lobes positions. Some parameters of valid configurations of the Costas-P4 combination, which were validated by simulations, are presented in Table 2. Parameters are chosen so as to achieve a Peak to Sidelobe Ratio PSLR of $20 \mathrm{~dB} \pm$, in the mainlobe region $|\tau| \leq t_{p}$ while keeping the bandwidth overflow $\frac{B}{\Delta f} \leq 5$. This reflects a significant lowering of grating lobes and could be convenient for many radar applications. In Table 2, P4 polyphase codes are used, since there is no restriction for their length ( $\mathrm{P} 4$ code of length $L$ is given by $\left[q_{l}=e^{\phi_{l}}\right]_{l=0: L-1}$ where $\phi_{l}=\frac{2 \pi}{L}(l) \frac{(l-L)}{2}$ ) and they show good autocorrelation properties that are needed to achieve targeted PSLR.
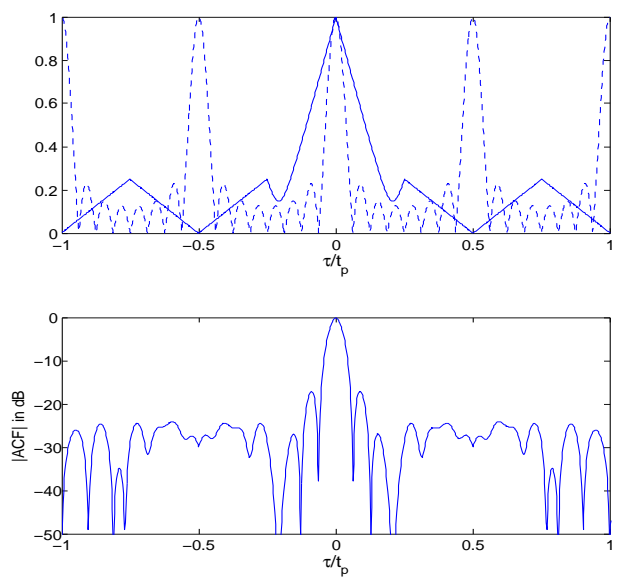

Figure 6: $\mathrm{ACF}$ of Phase coded Costas signal using $\mathrm{P} 3$ code with $\left(t_{p} \Delta f, L\right)=(2,4)$ top: continuous $\left|R_{1}\left(\frac{\tau}{t_{p}}\right)\right|$, dashed $\left|R_{2}\left(\frac{\tau}{t_{p}}\right)\right|$, bottom: $\left|R\left(\frac{\tau}{t_{p}}\right)\right|$

Table 2: Example of valid configurations for phase coded Costas signals (Costas code $M=8$ and $P 4$ phase codes)

\begin{tabular}{|c|c|c||c|c|c|}
\hline$t_{p} \Delta f$ & $t_{p} B_{p}(L)$ & $\frac{B_{p}}{\Delta f}$ & $t_{p} \Delta f$ & $t_{p} B_{p}(L)$ & $\frac{B_{p}}{\Delta f}$ \\
\hline 2 & 4 & 2 & 6 & 14 & 2.33 \\
2 & 8 & 4 & 7 & 9 & 1.28 \\
3 & 5 & 1.66 & 7 & 16 & 2.28 \\
3 & 9 & 3 & 8 & 12 & 1.5 \\
4 & 11 & 2.75 & 8 & 20 & 2.5 \\
4 & 14 & 3.5 & 9 & 12 & 1.33 \\
5 & 13 & 2.6 & 9 & 20 & 2.22 \\
5 & 18 & 3.6 & 10 & 14 & 1.4 \\
6 & 9 & 1.5 & 10 & 24 & 2.4 \\
\hline
\end{tabular}

We note that, for a fixed $t_{p} \Delta f$, the longer the code is, the better the lowering of grating lobes will be. This is due to the fact that, for most phase codes, the level of autocorrelation sidelobes decreases when their length is increased, which leads to a better lowering of grating lobes. However, such an increase in phase codes length could be more sophisticated for generation and bandwidth greedy. To design such signals, a large choice of phase codes is available in the literature. This gives more flexibility and diversity for an adaptation to a multi-user context by assigning a unique pulse code to each user. 
Touati, Nadiah; Tatkeu, Charles: Chonavel, Thierry; Rivenq, Atika:

Design and performances evaluation of new Costas-based radar waveforms with pulse coding diversity',

IET Radar, Sonar and Navigation, 2015, DOI: 10.1049/iet-rsn.2014.0450

\subsection{Costas signals with spectrally balanced signals}

Let us now focus on another diversity criterion to select codes. In a multi-user radar context, orthogonality of codes could be desirable in order to better distinguish between users and limit the interference. Hadamard codes are orthogonal codes intensely studied in coding theory. They are also much used in communication systems, mainly in CDMA systems[19]. Hadamard codes of length $N$ are a family of $N$ orthogonal binary codes with values +1 and -1 . Hadamard codes of lengths $N=2^{n}$ are easily built recursively: for $n=1$, the code family is given by the lines (or columns) of matrix $H_{2}=[1,-1 ; 1,1]$. Then for greater $n, H_{n}=H_{2} \otimes H_{n-1}$, where $\otimes$ denotes the Kronecker product [20]. However, these codes have poor correlation properties, making them poorly adapted to eliminate grating lobes. We are interested in a modified version of Walsh-Hadamard families where spectral efficiency and correlation properties are largely improved compared to those of original codes. In the following, the use of these codes in Costas pulses is proposed.

\subsubsection{Spectrally balanced Walsh-Hadamard codes}

In [21], the author proposes a balancing algorithm that transforms a family of orthogonal signals into another orthogonal family that spans the same subspace, so that the spectra of the signals in the new basis have very close spectra. This is achieved by iteratively balancing the energy of the basis signals in a set of prescribed subbands that span the whole signal bandwidth. To this end, a procedure that optimizes the energy balancing criterion is employed. It amounts to successively applying elementary rotations among the entries of the matrix associated to the discretized basis waveforms. An example of a result of this algorithm, when it is applied to $8 \times 8$ Walsh-Hadamard codes, is provided in Fig 7.
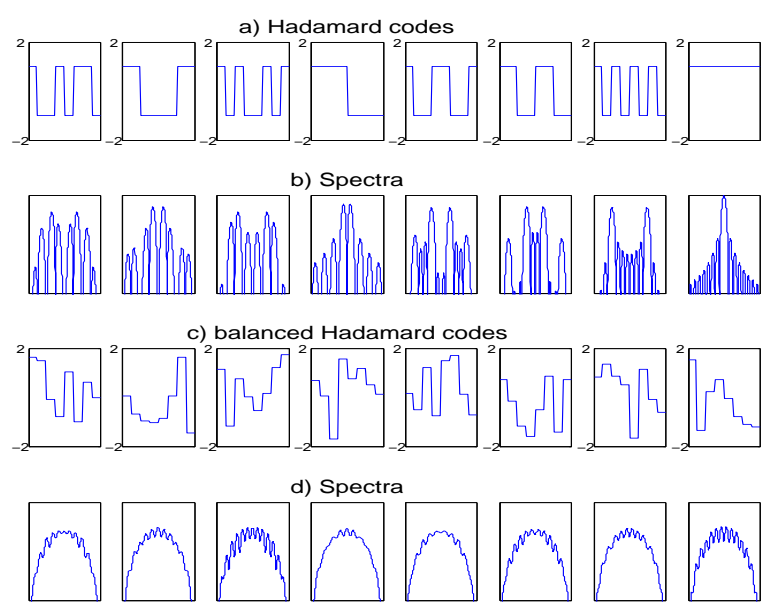

Figure 7: (a),(b) Walsh-Hadamard codes family $L=8$ and corresponding spectra (c),(d) spectrally balanced Walsh-Hadamard codes and corresponding spectra

As mentioned before, in addition to good spectral properties, correlation properties of original codes are largely improved. This is shown in Fig 8. This makes balanced Walsh-Hadamard codes suitable for Costas signals modification. An example of phase coded Costas signals involving a balanced Walsh-Hadamard code of length $L=8$ is shown in Fig 9. The low sidelobe level leads to a 
considerable lowering of grating lobes level. However, the limiting factor of spectrally balanced Walsh-Hadamard codes is that they can only be generated for lengths that are powers of $2\left(L=2^{k}\right)$. This restricts significantly the choice among phase codes and hence the $t_{p} B_{p}$ values. A more general approach is to use continuous sequences that can be generated for any bandwidth $B_{p}$. Good candidates for this approach are Slepian sequences.
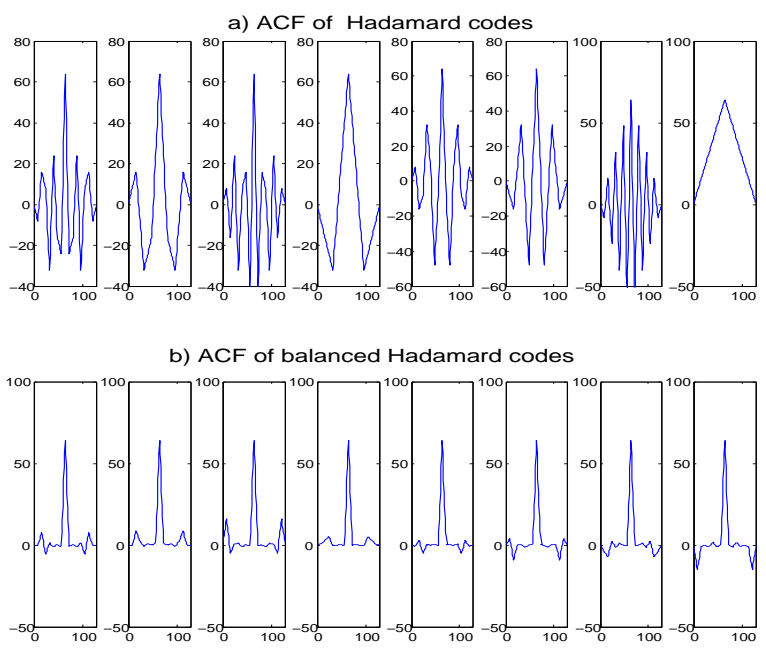

Figure 8: (a) ACFs of Walsh-Hadamard codes family $8 \times 8$ (b) ACFs of spectrally balanced Walsh-Hadamard codes $8 \times 8$
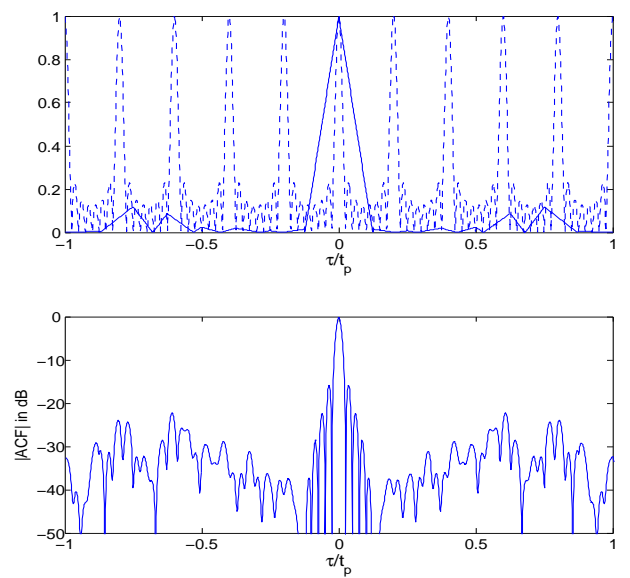

Figure 9: ACF of Phase coded Costas signal using a spectrally balanced Walsh-Hadamard code with $\left(t_{p} \Delta f, L\right)=(5,8)$ top: continuous $\left|R_{1}\left(\frac{\tau}{t_{p}}\right)\right|$, dashed $\left|R_{2}\left(\frac{\tau}{t_{p}}\right)\right|$, bottom: $\left|R\left(\frac{\tau}{t_{p}}\right)\right|$

\subsubsection{Spectrally balanced Slepian sequences}

The spectral concentration problem in Fourier analysis refers to finding a time sequence whose discrete Fourier transform is maximally localized on a given frequency interval. Slepian sequences are the solution to this problem. The advantage of these sequences is that they can be generated for any given bandwidth. However, as in the case of Walsh-Hadamard signals, these sequences have poor correlation properties, which makes them poorly adapted to radar applications. Spectrally balanced versions of Slepian sequences are interesting insofar as they combine Slepian sequences properties and improved correlation properties [21]. An example of Slepian 
sequences and spectrally balanced ones for a $t_{p} B_{p}=8$ is shown in Fig 10 and the improvement in ACFs before and after spectrum balancing is shown in Fig 11.

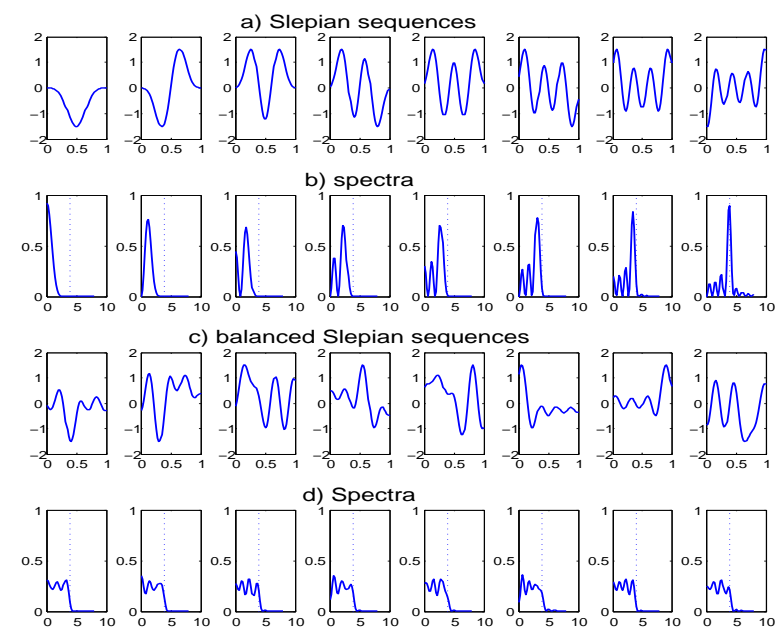

Figure 10: (a),(b)Slepian sequences family and corresponding spectra (c),(d) spectrally balanced Slepian sequences and corresponding spectra
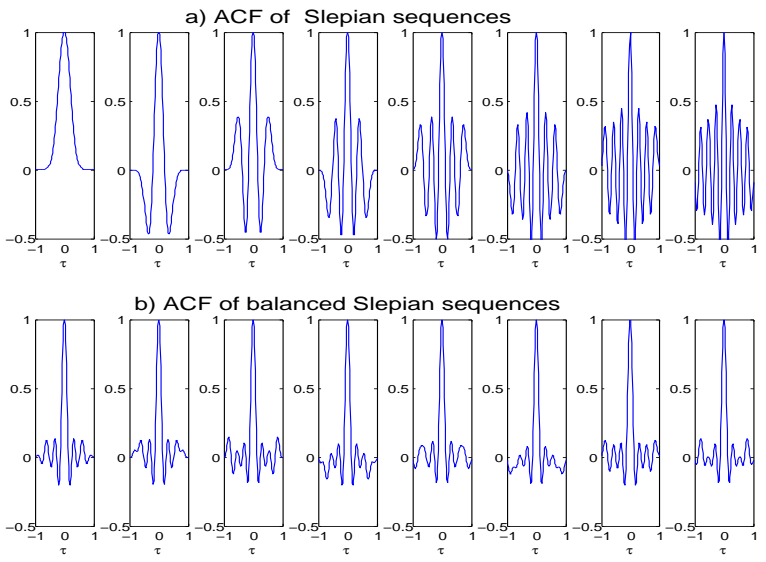

Figure 11: (a) ACFs of Slepian sequences (b) ACFs of spectrally balanced Slepian sequences

Using Slepian sequences to encode Costas pulses is interesting since the bandwidth of those sequences can be adapted to the $t_{p} \Delta f$ product of the Costas signal according to Eq 10. Furthermore, their number depends on the time-bandwidth product: $N=$ floor $\left(B_{p} t_{p}\right)$ different Slepian sequences can be generated. The case $t_{p} \Delta f=t_{p} B_{p}=5$ is illustrated in Fig 12. The first null of Slepian sequence ACF is positioned at the first grating lobe position and the other grating lobes are lowered according to Slepian $\mathrm{ACF}$ amplitude at their positions. Hence, it is preferable to use higher $t_{p} B_{p}$ product for Slepian waveforms to guarantee low Slepian ACF sidelobes and good grating lobes elimination. Then, to remain in accordance with the alignment of the first null with first grating lobe position, $t_{p} B_{p}$ must be chosen as a multiple of $t_{p} \Delta f$ : 


$$
t_{p} B_{p}=k \times t_{p} \Delta f, \quad k=1,2 \ldots
$$
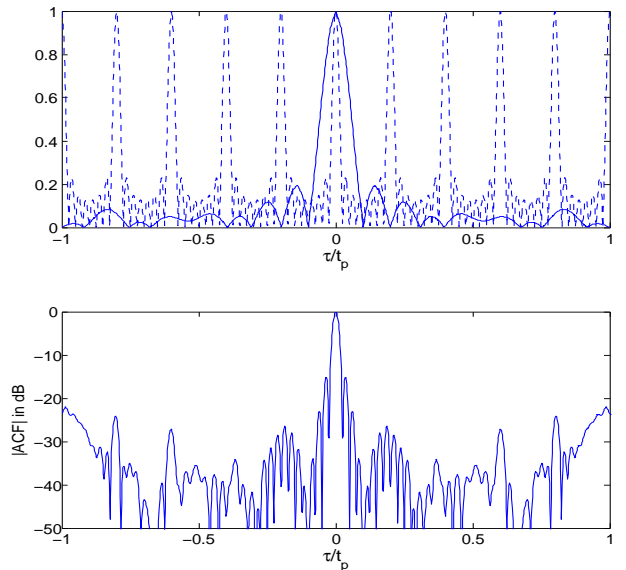

Figure 12: ACF of coded Costas signal using Slepian sequences with $\left(t_{p} \Delta f, t_{p} B_{p}\right)=(5,10)$ top: continuous $\left|R_{1}\left(\frac{\tau}{t_{p}}\right)\right|$, dashed $\left|R_{2}\left(\frac{\tau}{t_{p}}\right)\right|$, bottom: $\left|R\left(\frac{\tau}{t_{p}}\right)\right|$

Some valid configurations of the Costas-Slepian combination are presented in Table 3 . For a fixed $t_{p} \Delta f$, we note that the sidelobe level decreases when the sequence bandwidth is increased. This must be considered to select the best sidelobe level-bandwidth compromise. However, spectrally balanced Slepian sequences may show different ACF sidelobes from one sequence to another and this must also be taken into account when designing such signals. When compared to spectrally balanced Hadamard codes, Slepian sequences can lead to less efficient grating lobes lowering, due to a higher ACF sidelobes. However, they offer a large choice of sequences for any given $t_{p} B_{p}$.

Table 3: Example of valid configurations of Costas-Slepian combination (Costas code $M=8$ )

\begin{tabular}{|c|c|c||c|c|c|}
\hline$t_{p} \Delta f$ & $t_{p} B_{p}$ & $\frac{B_{p}}{\Delta f}$ & $t_{p} \Delta f$ & $t_{p} B_{p}$ & $\frac{B_{p}}{\Delta f}$ \\
\hline 2 & 2 & 1 & 6 & 12 & 2 \\
2 & 4 & 2 & 7 & 7 & 1 \\
3 & 3 & 1 & 7 & 14 & 2 \\
3 & 6 & 2 & 8 & 8 & 1 \\
4 & 4 & 1 & 8 & 16 & 2 \\
4 & 8 & 2 & 9 & 9 & 1 \\
5 & 5 & 1 & 9 & 18 & 2 \\
5 & 10 & 2 & 10 & 10 & 1 \\
6 & 6 & 1 & 10 & 20 & 2 \\
\hline
\end{tabular}

\subsection{Doubly coded Costas signals}

In this section, other Costas codes are used to encode the initial Costas pulses. The doubly coded Costas signal consists in replacing rectangular pulses by another Costas signal [22]. Indeed, under some conditions upon secondary Costas parameters, we have shown that such a double Costas coding approach can be efficient in eliminating grating lobes. The secondary Costas pulse duration is $t_{s}$ and frequency spacing is $\Delta f_{s}$. The complex envelope of the Costas pulse is given by: $A(t)=\sum_{l=0}^{L-1} v_{s}\left(t-l t_{s}\right)$ where $v_{s}(t)=\operatorname{Rect}\left(\frac{t}{t_{s}}\right) e^{j 2 \pi f_{l} t}$ and $f_{l}=l \Delta f_{s}$ are secondary Costas code elements. The only restriction on this design is that pulse duration 
Touati, Nadjah; Tatkeu, Charles; Chonavel, Thierry; Rivenq, Atika:

Design and performances evaluation of new Costas-based radar waveforms with pulse coding diversity',

IET Radar, Sonar and Navigation, 2015, DOI: 10.1049/iet-rsn.2014.0450

of main Costas $t_{p}$ is subdivided into $N$ sub-pulses of duration $t_{s}$ to contain the secondary Costas, i.e. $t_{p}=t_{s} \times N$. The bandwidth of the secondary Costas is $B_{p}=N \times \Delta f_{s}$ and hence $t_{p} B_{p}=N^{2} \times t_{s} \Delta f_{s}$. Then, the condition in Eq 10 becomes :

$$
N^{2} \times t_{s} \Delta f_{s} \geq t_{b} \Delta f
$$

When orthogonality condition of secondary Costas is not respected, namely $t_{s} \Delta f_{s} \geq 1$, ACF of secondary Costas signal $R_{1}\left(\frac{\tau}{t_{p}}\right)$ will also show its own grating lobes at $\tau_{s}\left(k_{1}\right)=\frac{k_{1}}{t_{s} \Delta f_{s}} t_{s}$ with $k_{1}=0, \pm 1 \cdots \pm$ floor $\left(t_{s} \Delta f_{s}\right)$. Hence, the design should consider this to avoid superposition of main and secondary Costas grating lobes, $\tau(k)$ and $\tau_{s}(k)$. In other terms, all positions of grating lobes should be different for all possible $\left(k, k_{1}\right)$ pairs, except at the origin (main lobe):

$$
\tau(k) \neq \tau_{s}\left(k_{1}\right) \Rightarrow \frac{k}{t_{p} \Delta f} t_{p} \neq \frac{k_{1}}{t_{s} \Delta f_{s}} t_{s}
$$

An example of a doubly coded Costas signal is given in Fig 13 where a secondary Costas code [1 243 ] with a $t_{s} \Delta f_{s}=0.78$ is used.
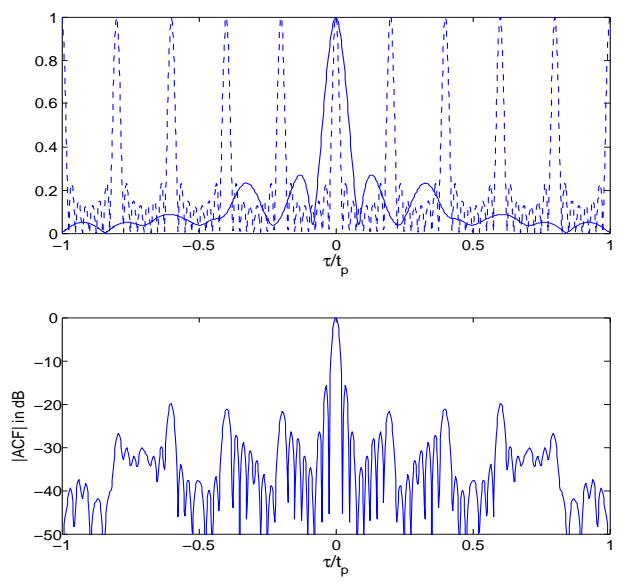

Figure 13: ACF of doubly coded Costas signal with $\left(M, N, t_{p} \Delta f, t_{s} \Delta f_{s}\right)=(8,4,5,0.78)$ top: continuous $\left|R_{1}\left(\frac{\tau}{t_{p}}\right)\right|$, dashed $\left|R_{2}\left(\frac{\tau}{t_{p}}\right)\right|$, bottom: $\left|R\left(\frac{\tau}{t_{p}}\right)\right|$

Some valid configurations of the Costas-Slepian combination are presented in Table 4 . We note that, when $t_{s} \Delta f_{s} \leq 1$, for a fixed secondary Costas size $N$, there are no secondary Costas grating lobes and the design is easier. However, when $t_{p} \Delta f$ increases, $t_{s} \Delta f_{s}$ must also be increased (for a fixed $N$ ), leading to the appearance of secondary Costas grating lobes. The secondary Costas size $N$ could also be varied in order to find the adequate $t_{p} B_{p}=N^{2} \times t_{s} \Delta f_{s}$. Such a design is original, since it combines waveforms of the same nature and the many variables of the system allow for better flexibility to adapt it.

\section{First sidelobe consideration}

In the previous sections, we focused on performances in terms of grating lobes lowering. Hence, we did not consider the first sidelobe, which is relatively high. This is a common problem in the standard LFM waveforms and Costas signals where this lobe is approximately $-13.7 d B$ below the mainlobe. For some radar applications, this may not be sufficient and it becomes annoying 
ouati, Nadjah; Tatkeu, Charles; Chonavel, Thierry; Rivenq, Atika:

Design and performances evaluation of new Costas-based radar waveforms with pulse coding diversity',

IET Radar, Sonar and Navigation, 2015, DOI: 10.1049/iet-rsn.2014.0450

Table 4: Example of valid configurations of Doubly coded Costas signals: Main Costas code $M=8$, secondary Costas code $N=4$

\begin{tabular}{|c|c|c|c||c|c|c|c|}
\hline$t_{p} \Delta f$ & $t_{s} \Delta f_{s}$ & $t_{p} B_{p}$ & $\frac{B_{p}}{\Delta f}$ & $t_{p} \Delta f$ & $t_{s} \Delta f_{s}$ & $t_{p} B_{p}$ & $\frac{B_{p}}{\Delta f}$ \\
\hline 2 & 0.5 & 8 & 4 & 6 & 1.2 & 19.2 & 3.2 \\
2 & 1 & 16 & 8 & 7 & 1 & 16 & 2.28 \\
3 & 0.55 & 8.8 & 2.93 & 7 & 1.3 & 20.8 & 2.97 \\
3 & 0.9 & 14.4 & 4.8 & 8 & 1.2 & 19.2 & 2.4 \\
4 & 0.75 & 12 & 3 & 8 & 1.4 & 26.9 & 3.3625 \\
4 & 1 & 16 & 4 & 9 & 1.3 & 20.8 & 2.31 \\
5 & 0.78 & 12.5 & 2.5 & 9 & 1.5 & 24 & 2.66 \\
5 & 1.1 & 17.6 & 3.52 & 10 & 1.5 & 24 & 2.4 \\
6 & 0.9 & 14.4 & 2.4 & 10 & 1.9 & 28.8 & 2.88 \\
\hline
\end{tabular}

for detection. Hence, it is proposed to extend the study to first sidelobe elimination, by positioning first null of Costas pulse ACF around the first sidelobe of $R_{2}\left(\frac{\tau}{t_{p}}\right)$ (instead of first grating lobe as for previous designs). High sidelobes are positioned between two successive grating lobes. They appear at local maxima of $R_{2}\left(\frac{\tau}{t_{p}}\right)$ (maxima of the numerator): $\tau_{l o c a l}(k)=\frac{(2 k+1)}{2 \times M \times t_{p} \Delta f} t_{p}$ where $k= \pm 1, \pm 2 \ldots$ Especially, if the first sidelobe $(k=1)$ has to be eliminated, the condition in Eq 10 becomes:

$$
\begin{aligned}
& \tau_{\text {first-null }} \leq \tau_{\text {local }}(1) \\
& \frac{1}{B_{p}} \leq \frac{3}{2 \times M \times t_{p} \Delta f} t_{p} \\
& t_{b} B_{p} \geq \frac{2}{3} \times M \times t_{p} \Delta f
\end{aligned}
$$

In the case of LFM pulse waveforms, the first null of the LFM can be exactly on the first sidelobe by setting $\pi B_{p} \frac{3}{2 \times M \Delta f}(1-$ $\left.\frac{3}{2 \times M \times t_{p} \Delta f}\right)=\pi$. Then:

$$
t_{p} B_{p}=\frac{4\left(M \times t_{p} \Delta f\right)^{2}}{3\left(2 \times M \times t_{p} \Delta f-3\right)}
$$

We show examples of modified Costas signals with respect to these conditions in Fig 14, 15, 16 and 17 for all previous designs. This case is bandwidth greedy since $t_{p} B_{p}$ is increased in order to eliminate the first sidelobe. The first sidelobe is considerably lowered, while the other sidelobes level is more or less unchanged.

\section{Performance comparison}

The overall bandwidth of the modified Costas signals is approximately $B_{\text {modified Costas }} \approx(M-1) \Delta f+B_{p}$ instead of $B_{\text {classic } \text { Costas }} \approx$ $M \Delta f$ for classic Costas signals. Hence, the time bandwidth product BT changes from $B T_{\text {classic } \text { Costas }}=M^{2}$ to:

$$
B T_{\text {modified Costas }} \approx M\left((M-1) \times t_{p} \Delta f+t_{p} B_{p}\right)
$$

Hence, modified versions introduce flexibility by adding other degrees of freedom to manipulate the time bandwidth product, which are $t_{p} \Delta f$ and $t_{p} B_{p}$. Furthermore, the target $B T$ product could be achieved without increasing the Costas codes size as in classic Costas. For example, for $M=8, t_{p} \Delta f=5$ and $t_{p} B_{p}=10$, we get $B T_{\text {modified Costas }} \approx 360$. To achieve the same $B T$ product with a classic Costas, a code of size $M=\sqrt{(360)} \approx 19$ is needed. In this part, the modified signals are compared 

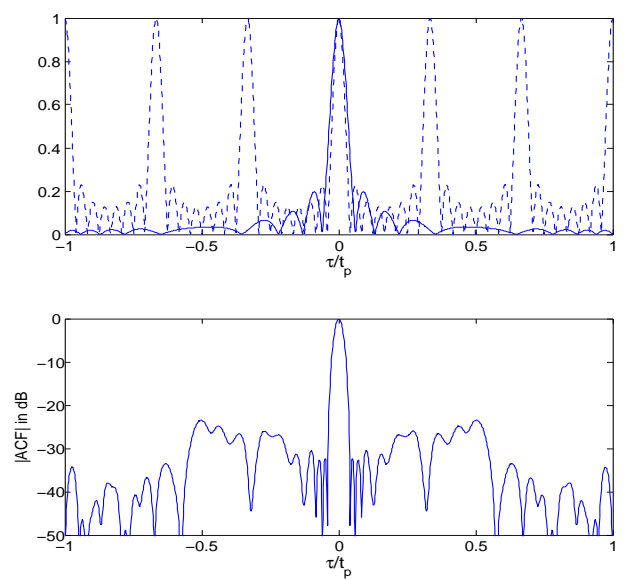

Figure 14: ACF of Costas-LFM signal with $\left(t_{p} \Delta f, t_{p} B_{p}\right)=(3,17.5)$, first sidelobe elimination top: continuous $\left|R_{1}\left(\frac{\tau}{t_{p}}\right)\right|$, dashed $\left|R_{2}\left(\frac{\tau}{t_{p}}\right)\right|$, bottom: $\left|R\left(\frac{\tau}{t_{p}}\right)\right|$
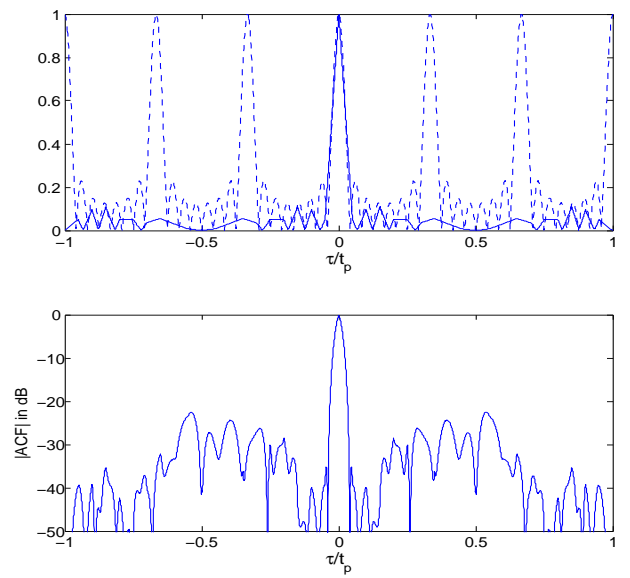

Figure 15: ACF of Costas-P4 signal with $\left(t_{p} \Delta f, L\right)=(3,20)$, first sidelobe elimination top: continuous $\left|R_{1}\left(\frac{\tau}{t_{p}}\right)\right|$, dashed $\left|R_{2}\left(\frac{\tau}{t_{p}}\right)\right|$, bottom: $\left|R\left(\frac{\tau}{t_{p}}\right)\right|$
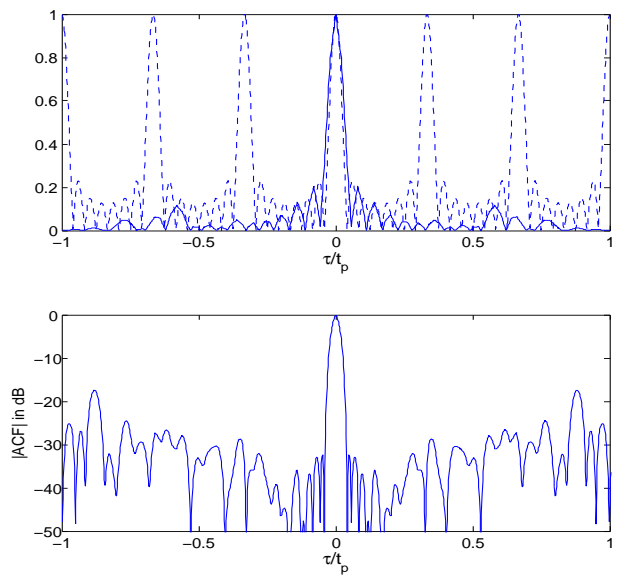

Figure 16: ACF of Costas-Slepian signal with $\left(t_{p} \Delta f, t_{p} B_{p}\right)=(3,18)$, first sidelobe elimination top: continuous $\left|R_{1}\left(\frac{\tau}{t_{p}}\right)\right|$, dashed $\left|R_{2}\left(\frac{\tau}{t_{p}}\right)\right|$, bottom: $\left|R\left(\frac{\tau}{t_{p}}\right)\right|$ 
Touati, Nadjah; Tatkeu, Charles; Chonavel, Thierry; Rivenq, Atika:

Design and performances evaluation of new Costas-based radar waveforms with pulse coding diversity',

IET Radar, Sonar and Navigation, 2015, DOI: 10.1049/iet-rsn.2014.0450
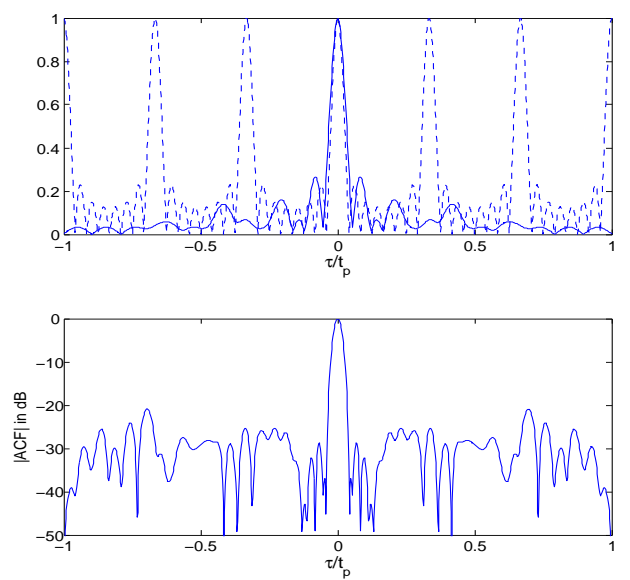

Figure 17: ACF of Doubly coded Costas signal with $\left(t_{p} \Delta f, t_{p} B_{p}\right)=(3,19)$, first sidelobe elimination top: continuous $\left|R_{1}\left(\frac{\tau}{t_{p}}\right)\right|$, dashed $\left|R_{2}\left(\frac{\tau}{t_{p}}\right)\right|$, bottom: $\left|R\left(\frac{\tau}{t_{p}}\right)\right|$

according to several criteria: bandwidth consumption, ambiguity function, delay-Doppler estimation accuracy, spectral properties and complexity .

\subsection{Bandwidth consideration}

How are the designs bandwidth greedy for a fixed target PSLR level?. Performances of proposed designs are discussed for a PSLR of $20 \mathrm{~dB} \pm 2 \mathrm{~dB}$, in the mainlobe region $|\tau| \leq t_{p}$ which reflects a significant lowering of grating lobes. Hence, we propose to calculate the mean of values of $t_{p} B_{p}$ in each proposed design, needed to achieve this PSLR. The max PSLR is also reported. The results are shown in Table 5.

Table 5: Comparison of mean values of $t_{p} B_{p}$ and achieved mean PSLR for the state-of-the-art method and the proposed designs

\begin{tabular}{|c|c||c|c|c|}
\hline Costas pulse nature & LFM & Phase code & Slepian & Costas code \\
\hline PSLR $(\mathrm{dB})($ mean) & 25.3 & 20.8 & 20.6 & 20.4 \\
\hline PSLR $(\mathrm{dB})(\mathrm{max})$ & 28 & 22.6 & 23.1 & 23.5 \\
\hline$t_{p} B_{p}$ (mean) & 17.9 & 12.9 & 9 & 17.7 \\
\hline$\frac{B_{p}}{\Delta f}$ (mean) & 3.07 & 2.33 & 1.5 & 3.31 \\
\hline
\end{tabular}

We can see that doubly coded Costas signals require more bandwidth than phase coded Costas signals and Slepian coded Costas signals. These last achieve the fixed PSLR with much less bandwidth. LFM approach cannot be compared very precisely to our approaches in terms of bandwidth consumption, since they do not have the same target PSLR. However, it can be compared to the doubly coded design since it has approximately the same bandwidth consumption. Using LFM pulses achieve a better PSLR (+5dB) than doubly coded Costas signals, with approximately the same $t_{p} B_{p}(\approx 18)$. However, the advantage of the double coding is the flexibility for an adaptation to a multi-user context, while this is less evident for LFM pulses. Indeed, the number of LFM pulses is limited to one for fixed $t_{p}$ and $B_{p}$ (or two if we consider an up and a down chirp). For some other families of pulse waveforms such as phase codes, Costas codes or Slepian sequences, several waveforms of the family could be generated for a fixed $t_{p}$ and $B_{p}$. We also note that for all designs, the PSLR can be about 3dB higher than the mean PSLR in the mainlobe region. In what follows, the ambiguity function is considered to discuss the sidelobe behavior and level out of the mainlobe region of the ACF. 
Touati, Nadjah; Tatkeu, Charles; Chonavel, Thierry; Rivenq, Atika:

Design and performances evaluation of new Costas-based radar waveforms with pulse coding diversity',

IET Radar, Sonar and Navigation, 2015, DOI: 10.1049/iet-rsn.2014.0450

\subsection{Ambiguity Function}

Theoretical delay and Doppler resolutions are inversely related to signal bandwidth and signal duration respectively: $\Delta \tau=\frac{1}{B}=$ $\frac{1}{(M-1) \Delta f+B_{p}}$ and $\Delta \nu=\frac{1}{T}=\frac{1}{M t_{p}}$. The AF allows us to verify this by evaluating the mainlobe Doppler and delay cuts widths. In the following, examples of AFs of modified Costas signals are proposed to give an overview about sidelobe behavior in all the delay-Doppler plane and not only in the ACF around the mainlobe, as previously done. Results are presented in Fig 18, Fig 19, Fig 20 and Fig 21. As can be seen on the ACF (zero Doppler cut), the sidelobe level for Costas-LFM signal is the lowest in the closest region to the mainlobe. However, the volume of the ACF is spread out to higher delays $|\tau| \geq t_{p}$ where it shows higher sidelobes compared to other modified signals that show sidelobes level more regularly distributed along the delay axis. Furthermore, most significant Doppler sidelobes are concentrated around the zero delay axis, for all modified signals. Indeed, the zero delay cut of the $\operatorname{AF} \chi(0, \nu)$, given in Eq 9, depends on zero delay cut of the pulse waveform $\chi_{A}(0, \nu)$ and a function that shows maxima at multiples of $\frac{1}{t_{p}}$ that can be seen as Doppler grating lobes. $\chi_{A}(0, \nu)$ is by definition, the Fourier transform of the squared magnitude of the pulse waveform $A(t)$. Hence, $\chi(0, \nu)$ depends heavily on amplitude properties of pulse waveforms. For constant amplitude signals it will be a sinc function and these lobes are significantly lowered. However, in the case of balanced signals, it will be different from one code (or a sequence) to another because of different amplitude properties. To avoid high Doppler sidelobes due to Doppler grating lobes, in this case, $\chi_{A}(0, \nu)$ the radar system should be designed in such a way that maximum detectable Doppler frequency remains smaller than the first Doppler grating lobe position, namely $\nu_{\max }<\frac{1}{t_{p}}$.

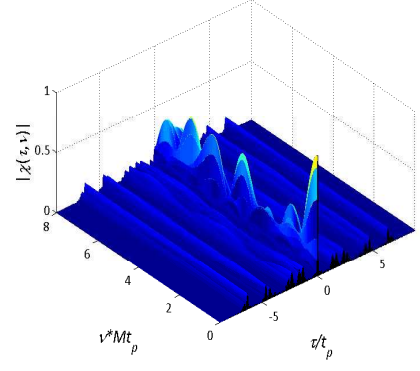

Figure 18: AF of the Costas-LFM signal with $\left(t_{p} \Delta f, t_{p} B_{p}\right)=(3,9)$

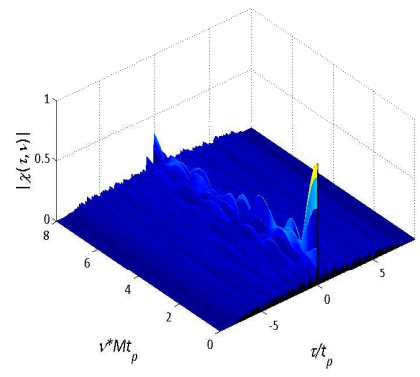

Figure 20: AF of the Costas-Slepian signal with $\left(t_{p} \Delta f, t_{p} B_{p}\right)=(3,9)$

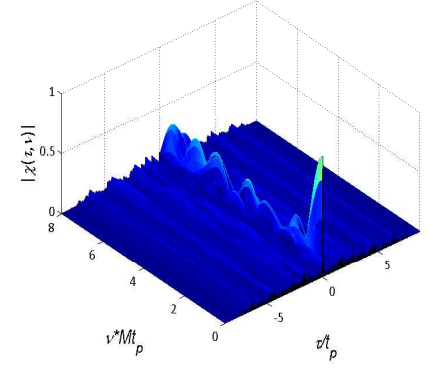

Figure 19: AF of the Costas-P4 signal with $\left(t_{p} \Delta f, t_{p} B_{p}\right)=(3,9)$

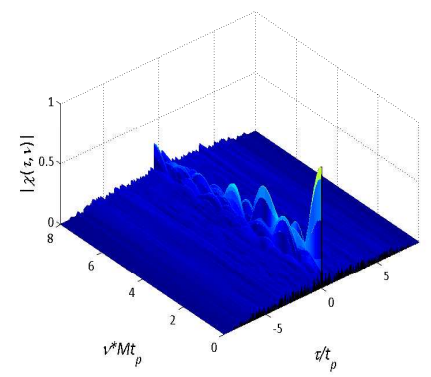

Figure 21: AF of the Doubly coded Costas signal with $\left(t_{p} \Delta f, t_{p} B_{p}\right)=(3,8.8)$ 
ouati, Nadiah; Tatkeu Charles; Chonavel, Thierry; Riveng Atika:

Design and performances evaluation of new Costas-based radar waveforms with pulse coding diversity',

IET Radar, Sonar and Navigation, 2015, DOI: 10.1049/iet-rsn.2014.0450

\subsection{Delay-Doppler estimation accuracy}

In this section, we evaluate delay-Doppler estimation accuracy using the Cramer-Rao Bound (CRB). Indeed, the CRB is a well known tool to get an indication about best achievable performance of a waveform in terms of delay-Doppler estimation, independent of the filtering algorithm. In the following, CRBs of modified signals are calculated and compared based on AF approximations. Indeed, the CRB is derived from the Fisher Inverse Matrix FIM $\mathbf{J}_{\mathbf{M}}^{-1}$ which is directly linked to the second derivatives of the squared magnitude of the ambiguity function $\Theta(\tau, \nu)=|\chi(\tau, \nu)|^{2}$ at $(\tau, \nu)=(0,0)$ [23].

$$
\begin{aligned}
& C R B(\tau)=\left[\mathbf{J}_{\mathbf{M}}^{-\mathbf{1}}(\tau, \nu)\right]_{(\mathbf{1}, \mathbf{1})} \\
& C R B(\nu)=\left[\mathbf{J}_{\mathbf{M}}^{-\mathbf{1}}(\tau, \nu)\right]_{(\mathbf{2 , 2})}
\end{aligned}
$$

where

$$
\mathbf{J}_{\mathbf{M}}(\tau, \nu)=-\mathbf{2} \mathbf{S N R}\left[\begin{array}{ll}
\frac{\partial^{2} \Theta(\tau, \nu)}{\partial \tau^{2}} & \frac{\partial^{2} \Theta(\tau, \nu)}{\partial \tau \partial \nu} \\
\frac{\partial^{2} \Theta(\tau, \nu)}{\partial \nu \partial \tau} & \frac{\partial^{2} \Theta(\tau, \nu)}{\partial \nu^{2}}
\end{array}\right]_{\tau=\mathbf{0}, \nu=\mathbf{0}}
$$

When analytical expressions of $\chi_{A}(\tau, \nu)$ in Eq 8, CRBs can be calculated directly derived. Analytical expressions are available for LFM waveforms and Costas codes but not for phase codes or Slepian sequences. Some approximations can be done to approximate the AF of such signals around the mainlobe which is sufficient to calculate the CRB. After some calculations, AF second derivatives can be derived for each signal. Results are shown in Table 6 for signals with bandwidth $B=100 \mathrm{MHz}$ and duration $T=2.4 \mu \mathrm{s}$ generated using a Costas code of size $M=8$. Here, CRBs are evaluated for a Signal to Noise Ratio SNR of $15 \mathrm{~dB}$ which is typical for radar applications. Standard deviations of the error on range $R$ and velocity $V, \sigma_{R}=\frac{c}{2} \sqrt{C R B(\tau)}$ and $\sigma_{V}=\frac{c}{2 f_{c}} \sqrt{C R B(\nu)}$ are also depicted when the central frequency is $\left(f_{c}=79 \mathrm{GHz}\right)$. We also propose to compare Range error bounds with matched filter MF results from simulations. Indeed, the MF is the maximum likelihood detection algorithm and its performances approach the CRBs for high $S N R$ s.

Table 6: CRBs and MF results for different modified Costas signals, when an $S N R=15 \mathrm{~dB}$ is considered

\begin{tabular}{|l|l|l|l|l|}
\hline Pulse waveform & LFM & P4 & Slepian & Costas \\
\hline$\left(t_{p} \Delta f, t_{p} B_{p}\right)$ & $(3,9)$ & $(3,9)$ & $(3,9)$ & $(3,8.8)$ \\
\hline$C R B(\tau)\left(s^{2}\right)$ & $4.5342 \times 10^{-19}$ & $4.4898 \times 10^{-19}$ & $4.5564 \times 10^{-19}$ & $3.5842 \times 10^{-19}$ \\
\hline$\sigma_{R}(m)$ & 0.1010 & 0.1005 & 0.1013 & 0.0898 \\
\hline$\sigma_{M F}(m)$ & 0.1259 & 0.1054 & 0.1353 & 0.1258 \\
\hline$C R B(\nu)\left(s^{-2}\right)$ & $7.5213 \times 10^{8}$ & $7.0879 \times 10^{8}$ & $7.2497 \times 10^{8}$ & $6.8088 \times 10^{8}$ \\
\hline$\sigma_{V}(m / s)$ & 52.07 & 50.55 & 51.12 & 49.54 \\
\hline
\end{tabular}

All signals have acceptable error standard deviation in range estimation. This could be verified by matched filter results which are very close to $\mathrm{CRBs}$ for all signals. Costas $-P 4$ achieve the minimal range error estimation using MF which is about $10.54 \mathrm{~cm}$. However, the error standard deviation in velocity estimation is relatively high and is not sufficient in practice for most radar applications. Indeed, this is due to the fact that only one signal is used here and the mainlobe is not sharp enough in the Doppler axis. In practice, a train of identical signals is used to increase signal duration which will lead to a sharpening of the mainlobe and hence reduced estimated Doppler errors. 
Touati, Nadjah; Tatkeu, Charles; Chonavel, Thierry; Rivenq, Atika:

Design and performances evaluation of new Costas-based radar waveforms with pulse coding diversity',

IET Radar, Sonar and Navigation, 2015, DOI: 10.1049/iet-rsn.2014.0450

\subsection{Spectral properties}

To compare spectral properties, we show example of spectra of discussed signals in Fig 22, Fig 23, Fig 24 and Fig 25. The CostasSlepian signals show remarkable spectral properties due to the spectrum balancing operated to the sequences. The LFM-Costas signals and doubly coded Costas signals also present good spectral properties with minimal out-of-band spectral sidelobes. However, the instantaneous phase switching due to the use of phase codes in the Costas-P4 signal causes extended spectral sidelobes. In practice, filters are used to cut off the spectrum tail which can slightly modify the final ACF. Other methods that propose implementing polyphase codes using a continuous phase modulation CPM in order to achieve spectral containment, could be used [24].

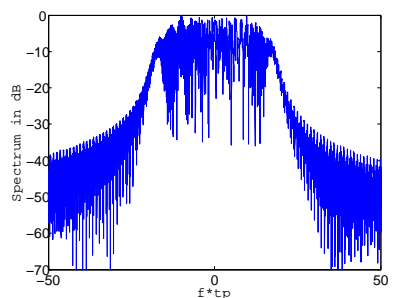

Figure 22: Spectrum of the Costas-LFM signal with $\left(t_{p} \Delta f, t_{p} B_{p}\right)=(4,11)$

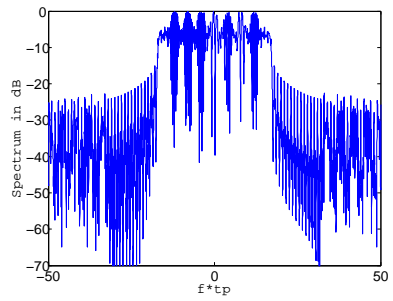

Figure 24: Spectrum of the Costas-Slepian signal with

$$
\left(t_{p} \Delta f, t_{p} B_{p}\right)=(4,12)
$$

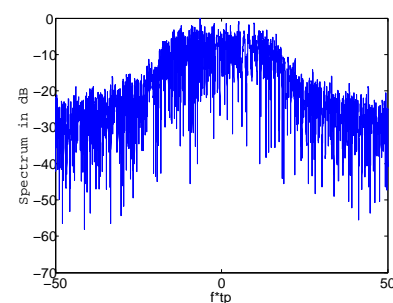

Figure 23: Spectrum of the Costas-P4 signal with $\left(t_{p} \Delta f, t_{p} B_{p}\right)=(4,11)$

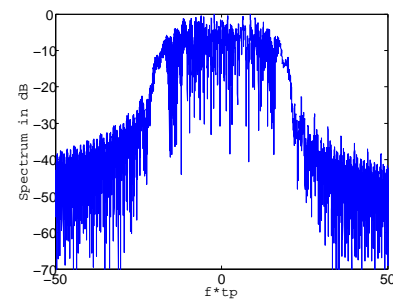

Figure 25: Spectrum of the Doubly coded Costas signal with $\left(t_{p} \Delta f, t_{p} B_{p}\right)=(4,12.5)$

\subsection{Complexity analysis}

A typical hardware architecture to generate proposed signals will be similar to an FHSS Frequency Hopping Spread Spectrum communication system, namely composed of a frequency hop generator involving a frequency synthesizer to select the Costas code $a_{m},(m=0: M-1)$ and generate modulated hopping waves at the frequencies $f_{m}$ and a pulse waveform generator to generate the pulse waveforms $A(t)$ (LFM, phase code, Slepian sequences or Costas code). The outputs of the pulse waveform generator and frequency hop generator are then fed to a modulator where the modulation is done by multiplying the pulse waveform and the modulated hopping waves. Hence, the complexity differences will occur at the waveform generator depending on the pulse nature. The analog generation of frequency modulated pulses, namely LFM and Costas pulses, is the easiest and could be achieved using a voltage-controlled oscillator VCO. However, digital approaches become tempting because of the substantial progress in the computational speed and the size of memory units. Hence, such signals could be generated digitally using Direct Digital Synthesis (DDS) units by supplying the needed frequency evolution at its input which will be linear for LFM pulses, and stepped for Costas. The complexity of phase codes generation depends, for its part, on the phase code nature. Polyphase codes are moderately more 
ouati. Nadiah: Tatkeu. Charles: Chonavel. Thierrv: Rivena. Atika

Design and performances evaluation of new Costas-based radar waveforms with pulse coding diversity',

IET Radar, Sonar and Navigation, 2015, DOI: 10.1049/iet-rsn.2014.0450

difficult to generate and need sophisticated units, such as DDS. For balanced Slepian sequences, the limiting factor is that they are not governed by a specific equation. Hence, the generation could be done exclusively using memory units to store directly those sequences instead of generating them each time.

\section{Conclusion}

This work illustrates several pulse compression techniques based on Costas signals. Methods for modifying Costas signals by widening frequency hops and replacing rectangular pulses by other waveforms that fall within this novel frequency spacing are proposed. This modification allow achieving BT product without increasing the size of the code. Furthermore, it leads to a lowering of autocorrelation grating lobes that appear when the frequency spacing is increased. The originality of the work consists in choosing variable waveforms for the Costas pulses, ranging from traditional signals, such as phase codes and Costas codes, to more sophisticated ones, such as spectrally balanced sequences. The nullification of the first sidelobe in the vicinity of the main lobe is discussed. It is shown that it is possible to suppress it as well as grating lobes, by a convenient choice of the bandwidth of the pulse waveform. A comparison between designs is also proposed to highlight the benefits and disadvantages of each approach. In future works, we will study another advantage, which is the flexibility of proposed designs to an adaptation to multi-user contexts that can help several radars to operate in close proximity.

\section{References}

[1] Skolnik, M.: Radar Handbook, Third Edition. McGraw-Hill Education, 2008.

[2] Levanon, N., Mozeson, E.: Radar signals. John Wiley and Sons, Inc, 2004.

[3] Costas, J.P.: A study of a class of detection waveforms having nearly ideal range ;doppler ambiguity properties,Proceedings of the IEEE, vol. 72, no. 8, pp. 996-1009, Aug.1984.

[4] Golomb, S., Taylor, H.: Constructions and properties of Costas arrays,Proceedings of the IEEE, vol. 72, no. 9, pp. 1143-1163, 1984.

[5] Beard, J., Russo, J.,Erickson, K., Monteleone, M., Wright, M.: Costas array generation and search methodology, IEEE Transactions on Aerospace and Electronic Systems, vol. 43, no. 2, pp. 522-538, 2007.

[6] Golomb, S.:The status of Costas array construction, in 40th Annual Conference on Information Sciences and Systems, March 2006, pp.522-524.

[7] Drakakis, K.: A review of Costas arrays,Journal of Applied Mathematics, vol. 2006, pp. 1-32, 2006.

[8] Freedman, A., Levanon, N.: Staggered Costas signals," IEEE Transactions on Aerospace and Electronic Systems, vol. AES-22, no. 6, pp. 695-702, Nov 1986. 
Design and performances evaluation of new Costas-based radar waveforms with pulse coding diversity',

IET Radar, Sonar and Navigation, 2015, DOI: 10.1049/iet-rsn.2014.0450

[9] Levanon, N., Mozeson, E.: Orthogonal train of modified Costas pulses, in Proceedings of the IEEE Radar Conference, April 2004, pp.255-259.

[10] Yang, H., Zhou, J., Wang, F., Zhang, Z.: Design and analysis of Costas/PSK RF stealth signal waveform, in IEEE CIE International Conference on Radar, 2011.

[11] Pace, P.E., Ng, C.Y.: Costas CW frequency hopping radar waveform: peak sidelobe improvement using Golay complementary sequences, Electronics Letters, vol. 46, no. 2, pp. 169-170, 2010.

[12] Lemieux, J., Ingels, F.: Analysis of FSK/PSK modulated radar signals using Costas arrays and complementary Welti codes, in Record of the IEEE International Radar Conference, 1990, pp. 589-594.

[13] Levanon, N., Mozeson, E.: Modified Costas signal, IEEE Transactions on Aerospace and Electronic Systems, vol. 40, no. 3, pp. 946-953, July.2004.

[14] Levanon, N., Mozeson, E.:Nullifying ACF grating lobes in stepped-frequency train of LFM pulses, IEEE Transactions on Aerospace and Electronic Systems, vol. 39, no. 2, pp. 694-703, 2003.

[15] Touati, N., Tatkeu, C., Chonavel, T., Rivenq, A.: Phase coded Costas signals for ambiguity function improvement and grating lobes suppression, in IEEE 78th Vehicular Technology Conference (VTC Fall), 2013, pp.1-5.

[16] Borwein, P., Kaltofen, E., Mossinghoff, M.: Irreducible polynomials and Barker sequences," ACM Commun. Comput. Algebra, vol. 41, no. 4, 2007.

[17] Lewis, B. L., Kretschmer, F.: A new class of polyphase pulse compression codes and techniques," IEEE Transactions on Aerospace and Electronic Systems, vol. AES-17, no. 3, pp. 364-372, 1981.

[18] Lewis, B. L., Kretschmer, F.: Linear frequency modulation derived polyphase pulse compression codes," IEEE Transactions on Aerospace and Electronic Systems, vol. AES-18, no. 5, pp. 637-641, 1982.

[19] Amadei, A., Manzoli, U., Merani, M.L.: On the assignment of Walsh and quasi-orthogonal codes in a multicarrier DSCDMA system with multiple classes of users, in IEEE Global Telecommunications Conference GLOBECOM '02. , Nov 2002, pp. 841-845.

[20] San Ling, C.: Coding Theory, A First Course, N. U. of Singapore, Ed. CAMBRIDGE University Press, 2004.

[21] Chonavel, T.: Orthogonal signals with jointly balanced spectra: Application to CDMA transmissions,EURASIP Journal on Wireless Communications and Networking, vol. 2011, no. 1, p. 176, 2011.

[22] Touati, N., Tatkeu, C., Chonavel, T., and Rivenq, A.: Doubly coded Costas signals for grating lobes mitigation, in IEEE 24th International Symposium on Personal Indoor and Mobile Radio Communications (PIMRC), Sept 2013, pp. 481-485.

[23] Gini, F., De Maio, A., Patton, L.: Waveform Design and Diversity for Advanced Radar Systems. The Institution of Engineering and Technology, London, United Kingdom, 2012. 


\section{Touati, Nadjah; Tatkeu, Charles; Chonavel, Thierry; Rivenq, Atika: \\ Design and performances evaluation of new Costas-based radar waveforms with pulse coding diversity',}

IET Radar, Sonar and Navigation, 2015, DOI: 10.1049/iet-rsn.2014.0450

[24] Blunt, S., Cook, M., Perrins, E., De Graaf, J.: CPM-based radar waveforms for efficiently bandlimiting a transmitted spectrum, in IEEE Radar Conference, May 2009, pp. 1-6. 